\title{
Deviant Peer Affiliation and Antisocial Behavior: Interaction with Monoamine Oxidase A (MAOA) Genotype
}

\author{
Steve S. Lee
}

Published online: 9 December 2010

(C) The Author(s) 2010. This article is published with open access at Springerlink.com

\begin{abstract}
Although genetic and environmental factors are separately implicated in the development of antisocial behavior (ASB), interactive models have emerged relatively recently, particularly those incorporating molecular genetic data. Using a large sample of male Caucasian adolescents and young adults from the National Longitudinal Study of Adolescent Health (Add Health), the association of deviant peer affiliation, the 30-base pair variable number tandem repeat polymorphism in promoter region of the monoamine oxidase-A (MAOA) gene, and their interaction, with antisocial behavior (ASB) was investigated. Weighted analyses accounting for over-sampling and clustering within schools as well as controlling for age and wave suggested that deviant peer affiliation and MAOA genotype were each significantly associated with levels of overt ASB across a 6-year period. Only deviant peer affiliation was significantly related to covert ASB, however. Additionally, there was evidence suggestive of a gene-environment interaction $(G \times E)$ where the influence of deviant peer affiliation on overt ASB was significantly stronger among individuals with the high-activity MAOA genotype than the low-activity genotype. MAOA was not significantly associated with deviant peer affiliation, thus strengthening the inference of $\mathrm{G} \times \mathrm{E}$ rather than gene-environment correlation (rGE). Different forms of gene-environment interplay and implications for future research on ASB are discussed.
\end{abstract}

Keywords Antisocial behavior · Longitudinal · Deviant peer affiliation $\cdot$ Gene-environment interaction

\footnotetext{
S. S. Lee $(\triangle)$

Department of Psychology, University of California, Los Angeles (UCLA),

1285 Franz Hall, Box 951563, Los Angeles, CA 90095-1563, USA

e-mail: stevelee@psych.ucla.edu
}

Antisocial behavior (ASB) consists of a diverse constellation of behaviors including violence and aggression, violating the rights/property of others, and breaking age-appropriate rules and norms (e.g., truancy). ASB is frequently persistent, resistant to treatment, and reliably associated with negative outcomes, including comorbidity (e.g., substance dependence, antisocial personality disorder), poor academic achievement, negative social relationships, and occupational instability (Loeber et al. 1998; Hinshaw and Lee 2003). The stability of overt/physical ASB across the lifespan approaches IQ, long considered one of the most stable individual attributes (Loeber and Stouthamer-Loeber 1998). Moreover, impairment related to ASB and externalizing behavior more broadly has historically constituted the most common referral for mental health services for American youth (Achenbach and Howell 1993; Kazdin 1995). Thus, ASB constitutes a significant public health problem and it remains an important scientific priority.

Broad characterizations of ASB, however, may betray important differences with respect to patterns of association, predictive validity, and etiological influences. A metaanalysis determined that two dimensions, consisting of overt and covert as well as destructive and non-destructive, described the underlying architecture of ASB (Frick et al. 1993). Using multi-method (i.e., laboratory, naturalistic observations) strategies of ASB assessment in school-aged boys, Hinshaw et al. (1995) found that covert ASB was independent of overt aggression and that covert ASB demonstrated discriminant and covergent validity with other constructs (i.e., attention-deficit/hyperactivity disorder (ADHD), child behavior problems). Indeed, the distinction between overt and covert ASB is evident early in development and persists through adolescence and young adulthood. Willoughby et al. (2001) found that overt aggression uniquely predicted other problems (e.g., class- 
room conflict, hyperactivity) relative to covert ASB. Next, a laboratory measure of childhood covert ASB significantly predicted adolescent psychopathology in two independent samples of boys and girls, controlling for overt aggression, ADHD, and peer rejection (Lee and Hinshaw 2004, 2006). Using latent class analysis (LCA) in a large study of adolescents and youth adults, covert ASB was empirically distinct from overt ASB, including patterns of sex differences in overt and covert ASB that were consistent with prevailing theories ( $\mathrm{Li}$ and Lee 2010). Finally, overt and covert ASB are separable with respect to genetic influences. Burt (2009) meta-analyzed twin studies and found that overt ASB was more heritable than covert ASB and that shared environmental influences were more salient for covert ASB than overt ASB. Finally, recent molecular genetic studies, across multiple designs (e.g., prospective longitudinal, clinical) and neurobiological systems (e.g., dopamine, serotonin) showed differential effects for overt vs. covert ASB ( $\mathrm{Li}$ and Lee 2010; Monuteaux et al. 2009). Overall, overt versus covert ASB is a meaningful distinction and one that should be prioritized in future studies, particularly genetic association studies which have largely ignored the distinction.

Deviant peer affiliation is a strong predictor of ASB from childhood through adolescence and early adulthood, across naturalistic and intervention designs (Dodge et al. 2006; Dishion and Patterson 2006). Deviant peer affiliation positively predicted childhood- and adolescent-onset trajectories of ASB, controlling for initial ASB, suggesting its centrality in the emergence, maintenance, and escalation of ASB over time (Gordon et al. 2004; LaCourse et al. 2006). Among 267 boys and girls entering kindergarten, deviant peer affiliation significantly predicted overt and covert conduct problems across home, school, and playground contexts (Snyder et al. 2005). Similarly, Monahan et al. (2009) demonstrated that deviant peer association prospectively predicted ASB in a sample of 1,354 youth (ages 14 to 22) with incarceration histories. Finally, Fergusson et al. (2002) reported that among 1,265 children, followed prospectively into young adulthood, deviant peer affiliation predicted diverse negative outcomes (i.e., substance problems, violence, property crime), although the magnitude of the association was stronger in adolescents (14-15 yearsold) than young adults (20-21 years-old). Thus, deviant peer affiliation is a risk factor for ASB and these influences have been suggested across development.

Despite the persuasive evidence on the association of deviant peer affiliation and ASB, not all youth with deviant peers engage in ASB or equally affected by deviant peers. In a study of 6th grade children followed prospectively into adolescence, individual differences in effortful and inhibitory control moderated the influence of deviant peer affiliation on ASB. Specifically, deviant peer affiliation significantly predicted ASB only among the subgroup of youth with poor self-regulation. Among youth with welldeveloped self-regulation, deviant peer affiliation did not predict ASB (Gardner et al. 2004). In a prospective study of school-aged children, age 14 deviant peer affiliation significantly predicted age 16 ASB even when age 14 ASB was controlled. However, adolescents with heightened reward sensitivity were particularly susceptible to peer deviance (Goodnight et al. 2006). Other studies suggest individual differences in response inhibition, coupled with deviant peer affiliation, may be particularly predictive of ASB (Kendler et al. 2008) whereas youth with a positive emotional attachment to their parents prospectively reported lower levels of ASB in the presence of deviant peer affiliation (Vitaro et al. 2000). Given that all youth are not equally susceptible to deviant peer influences, there is a need to identify moderators of deviant peer effects on ASB. The detection of subgroups of youth who may be particularly vulnerable to deviant peers may benefit from targeted intervention and prevention efforts.

One important class of moderators that has yet to be thoroughly explored is genetic liability. Genetic susceptibility may provide additional traction on the conditions in which deviant peer affiliation predicts ASB. Among 3596 year-old twins, deviant peer affiliation and genetic risk (defined by the co-twin's aggression and the pair's zygosity) were each independently associated with aggression. However, the influence of deviant peers was significantly stronger among genetically vulnerable children, suggesting a gene-environment interaction $(G \times E)$ (van Lier et al. 2007). Similarly, in a study of 4067 year-old twins (Brendgen et al. 2008), variability in physical aggression was significantly explained by the interaction between friends' aggression and child genetic vulnerability (based on zygosity and co-twin's aggression). Importantly, the putative $\mathrm{G} \times \mathrm{E}$ was not significant for social aggression, suggesting that $G \times E$ effects may be specific to physical aggression (e.g., social aggression is more strongly correlated with popularity and prosocial attention) (Brendgen et al. 2008) and different forms of ASB more generally (e.g., proactive vs. reactive aggression). These studies collectively underscore the plausibility that genetic factors change the nature of deviant peer affiliation influences on ASB and the importance of specifying subtypes of ASB.

However, the mechanisms mediating deviant peer affiliation and ASB have not been ascertained. Deviant peer affiliation may provide an ideal context to engage in ASB because of positive reinforcement (i.e., peer approval) and modeling or genetic mediation. Kendler et al. (2007) uncovered genetic effects on peer group deviance (Kendler et al. 2007) and a population-based study estimated that $64 \%$ of adolescents' exposure to peers who smoke or drank was genetically influenced (Cleveland et al. 2005). Passive, 
evocative, and active gene-environment correlations (rGE) raise the possibility that deviant peer affiliation effects and ASB may be partially explained by genetic effects (Jaffee and Price 2007; Monahan et al. 2009). Thus, latent genetic factors covarying with aspects of the environment suggest that molecular genetic assays constitute an important direction for future research.

Despite the value of traditional behavior genetic designs, they are anonymous in that they do not identify specific genetic variants, therefore preventing inferences about the underlying biology regulated by genetic variation. However, molecular genetic assays directly test genomic variation and once they are replicated, they provide a strong empirical basis to explore the neurobiological consequences of genetic differences (e.g., neural recruitment/activation). One strong candidate for ASB is the monoamine oxidase-A gene (MAOA) given that the enzyme associated with this gene plays a significant role in the catabolism of synaptic catecholamines such as dopamine, serotonin, and norepinepherine. The MAOA gene has been mapped to chromosome Xp11.23Xp11.4 (Ozelius et al. 1988). In particular, the 30-base pair (bp) variable number tandem repeat (VNTR) polymorphism in the promoter region of MAOA has garnered significant interest because long repeat sequences of this functional polymorphism result in greater transcriptional efficiency than short repeat sequences (Sabol et al. 1998). Based on evidence from transgenic mice models (Cases et al. 1995) and from a carefully characterized simplex family of affected males and carriers with a point mutation in the MAOA gene (Brunner et al. 1993), monoamine may be particularly salient for ASB and related phenotypes.

Studies of MAOA and ASB have been inconsistent, however. In a sample of nearly 1,000 7 year-old children, low-activity MAOA genotypes significantly predicted ASB, ADHD, and an aggregate measure of mental health problems, but they were unrelated to emotional problems (Kim-Cohen et al. 2006). Main effects for the high-activity MAOA genotype and aggression were reported in a small sample of psychiatrically-referred boys (mean $=9.2$ years) (Beitchman et al. 2004). Null findings for MAOA and ASB were reported among 247 adolescent boys (12-18 years) referred for intensive day or residential treatment (Young et al. 2006). In a sample of 277 11-15 year-old Caucasian boys, Huizinga et al. (2006) found no association of MAOA and ASB (e.g., conduct disorder (CD), arrests for violence). And finally, based on 774 Caucasian adolescents and young adults from the National Longitudinal Study of Adolescent Health, MAOA was unrelated to self-reported ASB (Haberstick et al. 2005). It is important to note that all of these studies were cross-sectional, with the exception of Haberstick et al. (2005), who collapsed multi-occasion measures of ASB into a single composite. This is a limitation given that age-related changes in ASB are reliable and they may misrepresent differences in underlying mechanisms and prognosis (Hinshaw and Lee 2003). Lahey et al. (2002) demonstrated that boys with persistent conduct disorder (CD) had the highest levels of comorbid anxiety, depression, ADHD, and oppositional defiant disorder (ODD) than youth with less stable CD. Similarly, more than $90 \%$ of $7-15$ year-old girls had an age of onset for $\mathrm{CD}$ prior to age 10 , with a single common pathway to $\mathrm{CD}$ consisting of the intensification of symptoms first evident in childhood rather than an abrupt onset in adolescence (Keenan et al. 2010). Thus, prospective longitudinal studies are necessary to delineate unique pathways and patterns of association.

In addition to psychopathology, MAOA variants are significantly related to potential endophenotypes for ASB (i.e., intermediate phenotypes between etiology and explicit behavior) (Gottesman and Gould 2003). For example, the 30-base pair (bp) VNTR in the promoter region of MAOA was significantly associated with personality traits relevant to ASB (i.e., harm avoidance, reward dependence) (Buckholtz et al. 2008) and brain MAOA activity measured in vivo was associated with trait measures of aggression (Alia-Klein et al. 2008). In addition to main effects, MAOA has been implicated in interactive models for ASB across social environments. Among rhesus monkeys, the influence of rearing experiences on measures of aggression was stronger among monkeys with the low-activity 7-repeat allele of the MAOA genotype (Karere et al. 2009). Levels of adolescent and adult ASB among children with severe exposure to maltreatment were significantly higher in individuals with the low-activity MAOA genotypes (i.e., 3-repeat and 5repeat) versus high-activity genotypes (i.e., 3.5- and 4repeat) differed by MAOA genotype (Caspi et al. 2002). Subsequent validation using meta-analysis with low-activity MAOA genotypes defined as 2-, 3-, and 5-repeat alleles has also been reported (Kim-Cohen et al. 2006). MAOA genotype also moderated the teratogenic consequences of prenatal exposure to nicotine where boys with the lowactivity MAOA genotypes (i.e., 3-, 5-repeat) and girls with the high-activity MAOA genotypes (i.e., 3.5-, 4-repeat) exhibiting more adolescent $\mathrm{CD}$ than youth with different genotypes (Wakschlag et al. 2010).

Although the precise explanatory factors for MAOA increasing susceptibility to negative environments are unknown, MAOA genotype has been implicated in the 'socioaffective scaffold' that regulates social evaluation, decision-making, and emotion regulation. That is, individuals with particular genotypes possess compromised "neural equipment" (p. 127) that sensitizes them to negative social and interpersonal experiences that propel the development of ASB (and aggressive forms in particular) (Buckholtz and Meyer-Lindenberg 2008). Thus, in the context of deviant peer affiliation, individuals with the low- 
activity MAOA genotype may be differentially sensitive to the deviant peer effects, given disruptions to socioemotional functioning, particularly with respect to decision making in a social context. Together with evidence that adolescent-limited offenders, whose ASB is less heritable, largely covert (i.e., status offenses), and driven substantially by peer networks (Moffitt et al. 2001), overt ASB should be more sensitive to MAOA variation than covert ASB. To test the specificity of MAOA and its association with aggression and violence (Buckholtz and Meyer-Lindenberg 2008), studies must separately assess overt and covert ASB. Collectively, these studies suggest that the low-activity MAOA genotype is a plausible candidate gene for ASB (and overt ASB specifically), particularly in the context of interactions with variation in negative social experiences, including deviant peer affiliation.

Overall, there are three important features of this study that collectively represent an advance over previous studies. First, this sample consists of adolescents and adults prospectively followed across approximately six years. This approach is necessary to explore the discriminant validity of behaviorally defined syndromes and disorders (Eley, Lichtenstein, and Moffitt 2003) and it is consistent with recommendations in the area of $\mathrm{G} \times \mathrm{E}$ for peer influences and aggression (van Lier et al. 2007; Brendgen et al. 2008). This is also important given that repeated measures designs improve the precision of phenotype measurement and thereby enhance statistical power (Schmitz et al. 1998), a longstanding challenge in the genetic epidemiology of complex phenotypes (Risch and Merikangas 1996). Second, unlike previous studies that were limited to the anonymous approach of traditional behavior genetic studies, this study tested the contribution of MAO-A genotype, a functional variant affecting neural activation and sensitivity to environments. Third, this study simultaneously integrated a developmentally-informative and geneticallysensitive design with separate measures of overt and covert ASB in a largely adolescent sample, given that overt and covert ASB possess unique patterns of association with other domains of influence (e.g., heritability, family factors).

Although this is not a representative sample, the participants were not clinic-referred, thereby avoiding the biases of such samples, including high comorbidity and early onset of problems and impairment (Goodman et al. 1997). To review, the goal of the study was two-fold: (1) To test the independent association of deviant peer affiliation and MAOA genotype on self-reported ASB collected prospectively across three separate assessments in adolescence and early adulthood; and (2) To test MAOA as a potential moderator of deviant peer affiliation effects on overt and covert ASB. Based on the extant literature, we hypothesize that deviant peer affiliation will positively predict overt and covert ASB whereas MAOA genotype (i.e., low-activity polymorphisms) will positively predict overt ASB only. Furthermore, we expect that the effects of deviant peer affiliation on ASB will be stronger in the lowversus high-activity MAOA genotype (i.e., gene-environment interaction).

\section{Method}

\section{Participants}

The National Longitudinal Study of Adolescent Health (Add Health) ascertained a stratified random sample of youth from U.S. high schools, with oversamples based on race-ethnicity. In Wave I, 20,745 adolescents (grades 7-12, ages 12-20 years during the 1994-1995 school year; 47.5\% male) were interviewed. One year later, in Wave II, approximately 15,000 of the same youth were assessed during in-home interviews. Approximately 5 years later, an embedded genetic sample, consisting of all full siblings and twins, was asked to provide saliva for genetic analyses in Wave III $(n=3,787)$. The subjects at Wave III had a mean age of 22.4 years $(S D=$ 1.7). Analyses were limited to Caucasian males to enhance comparisons with existing studies of MAOA and ASB in the literature (Caspi et al. 2002; Edwards et al. 2010; Foley et al. 2004). Because MAOA expression in women cannot be unambiguously determined (Carrel and Willard 2005), experts recommend that analyses of MAOA be limited to men (Kim-Cohen et al. 2006). Analyses were conducted on the 670,631 , and 672 youth with available data at Wave I, II, and III, respectively. Demographic descriptions of the sample are provided in Table 1.

These data are contractually available to certified researchers and details are available at http://www.cpc.unc.edu/projects/ addhealth. Parental consent was required to allow students to participate in the study. Unless otherwise directed by the school, passive consent forms were utilized. In other words, it

Table 1 Means and standard deviations of demographic variables by MAOA genotype

\begin{tabular}{|c|c|c|c|c|}
\hline \multirow[t]{2}{*}{ Measure } & \multicolumn{2}{|c|}{$\begin{array}{l}\text { High activity MAO } \\
(n=419)\end{array}$} & \multicolumn{2}{|c|}{$\begin{array}{l}\text { Low activity MAO } \\
(n=253)\end{array}$} \\
\hline & M & SD & M & SD \\
\hline Age & 15.7 & 1.6 & 15.6 & 1.7 \\
\hline Family income & 49.2 & 44.4 & 53.4 & 59.3 \\
\hline DPA & 0.23 & 0.25 & 0.20 & 0.25 \\
\hline Overt ASB & 0.34 & 0.92 & 0.38 & 0.94 \\
\hline Covert ASB & 0.69 & 1.47 & 0.89 & 1.85 \\
\hline
\end{tabular}

Overt and covert ASB each reflect behavior across three waves of data collection

$D P A$ deviant peer affiliation; $A S B$ antisocial behavior 
was assumed that a parent granted permission unless the form was returned with a signature that indicated otherwise, although some schools required active consent forms. Written informed consent was obtained from the parent or legal guardian and the adolescent for the in-home interviews.

\section{Measures}

\section{Antisocial Behavior}

At each of the three assessments, conducted during in-home interviews, individuals reported the frequency of 10 types of ASB, including fighting resulting in harm, group fighting, assault with a weapon, vandalism, selling marijuana, breaking and entering, and stealing. Response options for the ASB items were as follows: 0 (none), 1 or 2 times, 3 or 4 times, or $5+$ times. We summed the 10 behaviors at each assessment and analyzed the data as three separate repeated measures. Standardized Cronbach alphas were $0.89,0.77$, and 0.86 for the three ASB measures at waves 1-3, respectively. Although these ASB items are not directly derived from DSM-IV based inventories or interviews, they do share similar properties to $C D$ including separate measures of overt ASB (e.g., fighting) and covert ASB (e.g., theft) and they refer to a similar period of time (i.e., behavior in the previous 12 months).

\section{Deviant Peer Affiliation}

At Wave 1, youth were asked how many of their three closest friends smoked at least one cigarette each day, smoked marijuana at least 1 time each month, and drank alcohol at least 1 time each month. Participants were also asked about how often they took part in a fight where his/her group of friends fought against another group of people (past 12 months). Based on an ordinal scale, participants indicated whether they fought with their peers against other youth $0,1-$ 2, 3-4, or more than 5 times. These four variables (smoking, marijuana, alcohol, group violence) were standardized via zscores and then averaged to create an overall measure of deviant peer affiliation. The standardized Cronbach alpha for the composite measure was 0.64 .

\section{Genotyping}

At Wave III, genomic DNA was isolated from buccal cells using standard methods. MAOA genotype was assayed by a minor modification of a published method. Primer sequences for the $30 \mathrm{bp}$ VNTR in the promoter region of the MAOA were: forward, 5'ACAGCCTGACCG-TGGAGAAG-3' (fluorescently labeled), and reverse, 5'GAACGTGACGCTCCATTCGGA-3'. Products of this reaction included five possible fragment sizes that included 291, 321, 336, 351, and 381 bps. Genotypes were scored independently by two individuals. Following the procedures outlined previously (Edwards et al. 2010; Foley et al. 2004; Kim-Cohen et al. 2006), 336 (3.5-repeat) and 351 (4repeat) polymorphisms were coded into a high-activity group ( $n=419)$ and 291 (2-repeat), 321 (3-repeat), and 381 (5-repeat) polymorphisms were coded into a low-activity group $(n=253)$. These groups are associated with more and less transcriptional efficiency, respectively. The genotype distribution was as follows: 291/291 $(n=1), 321 / 321(n=$ 238), 336/336 $(n=7), 351 / 351(n=412)$, and 381/381 $(n=$ 14) (see Table 1 summarizing demographic and behavioral characteristics of MAOA genotype groups).

\section{Statistical Analyses}

Weighted descriptive analyses were conducted to take clustering within schools, oversampling, and the complex survey design into account. Tests of the association of genotype and deviant peer affiliation with overt and covert ASB at each wave were conducted using log-linear regression in generalized estimating equations (GEE) in SAS GENMOD, specifying Poisson distributions and an autoregressive correlation structure. GEE estimates change over time at the population level rather than at the individual or cluster-specific level. Because GEE estimate means in each assessment from available data, they accommodate missing data well, especially when retention is high, as in the present study. GEE is superior to generalized linear models for several reasons (Hanley et al. 2003): (1) more reliable standard errors and improved confidence intervals; (2) maximum flexibility to specify the working correlation matrix (e.g., exchangeable, unstructured), although auto-regressive is featured here given the clustered nature of the observations over time; (3) even if the matrix is misspecified, parameter estimates and variance estimators are generally stable; and (4) the use of an empirical or robust variance estimator is appropriate because one is typically unaware of the correct covariance structure.

Youth with available genotype data did not differ in total ASB from the youth in the Wave I sample who were not genotyped $[\mathrm{F}(1,20311)=2.95, p=0.09$, respectively $]$, although there were marginally fewer boys $(47.8 \%$ vs. $52.2 \%), \chi^{2}=3.45, p=0.06$ and significantly fewer African Americans ( $18 \%$ vs. $22.9 \%), \chi^{2}=29.8, d f=1, p<0.001$ and Hispanics among genotyped youth $(12.2 \%$ vs. $17.3 \%), \chi^{2}=$ 12.8, $d f=1, p<0.001$ than in the overall sample. Because MAOA genotypes were non-randomly distributed by raceethnicity $\left(\chi^{2}=78.4, d f=12, p<0.0001\right)$, a necessary condition for population stratification (Hutchison et al. 2004), only data from Caucasian males were analyzed to avoid spurious findings that are the result of allelic differences in ancestral populations. Finally, to further characterize the 
sample of Caucasian males with MAOA genotypes featured in these analyses against the overall genotyped sample, comparisons on age and family income were conducted. No significant differences were observed between the two groups of youth for age $\left(\chi^{2}=4.84, d f=8, p=0.78\right)$. However, youth in the current study came from families with significantly higher incomes than the remaining genotyped sample $(t(428)=4.28, p<0.0001)$. Although family income was originally included as a covariate to account for this difference, its inclusion did not meaningfully change the results presented below. Consequently, it was not retained as a covariate.

\section{Results}

Overt ASB: MAOA Genotype, Deviant Peer Affiliation, and their Interaction $(\mathrm{G} \times \mathrm{E})$

Total counts of ASB may betray disparate influences underlying overt/aggressive and covert/non-aggressive forms, including genetic influences and gene-environment interplay (Burt 2009; Edelbrock et al. 1995). To ascertain the specificity of deviant peer affiliation and MAOA influences on diverse forms of ASB, generalized estimating equations (GEE) were modeled separately for five overt ASB items, controlling for age and wave (see Table 2, Model 1 for a model summary of parameters for covariates and main effects only). In the fully saturated model, consisting of covariates (i.e., age and wave), main effects, and the deviant peer affiliation $\times$ MAOA genotype interaction term (see Table 2, Model 2), there was a significant effect of MAOA genotype $(\beta=-0.56, z=-2.19$, $p<0.05)$ and deviant peer affiliation $(\beta=1.26, z=2.28, p<$ $0.05)$. This suggests that individuals with the low-activity

Table 2 Generalized estimating equation (GEE) predicting overt antisocial behavior (ASB)

\begin{tabular}{lrrl}
\hline Variables & $\beta$ & \multicolumn{1}{l}{$z$} & $p<$ value \\
\hline Model 1 & & & \\
Age & -0.19 & -3.28 & 0.001 \\
Wave & -0.06 & -1.90 & 0.05 \\
Deviant peer affiliation (DPA) & 2.28 & 7.58 & 0.0001 \\
MAOA genotype & -0.03 & -0.13 & 0.89 \\
Model 2 & & & \\
Age & -0.19 & -3.46 & 0.0001 \\
Wave & -0.06 & -1.91 & 0.05 \\
Deviant peer affiliation (DPA) & 1.26 & 2.28 & 0.05 \\
MAOA genotype & -0.56 & -2.19 & 0.05 \\
Deviant peer $\times$ MAOA interaction & 1.57 & 2.67 & 0.01 \\
\hline
\end{tabular}

MAOA genotype, which is associated with poor transcriptional efficiency of monoamine, reported significantly more overt ASB across 6 years than those in the high-activity group. The adjusted means and standard errors for the genotype groups for overt ASB were $M=0.27, S E=0.11$ and $M=0.34, S E=0.16$ for the high- and low-activity groups, respectively (see Table 2, Model 2).

A potential gene-environment interaction $(\mathrm{G} \times \mathrm{E})$ was explored by testing whether the association of deviant peer affiliation effects and ASB were moderated by MAOA genotype. This model controlled for age and wave, included the main effects for deviant peer affiliation and MAOA genotype, and included a separate interaction term. There was evidence consistent with a deviant peer affiliation $\times$ MAOA interaction for overt ASB $(\beta=1.57, z=2.67, p<0.01)$ (see Table 2, Model 2). That is, deviant peer affiliation predicted overt ASB more strongly among individuals with the high-activity MAOA genotype (see Fig. 1). Among youth with the low-activity MAOA genotype, deviant peer affiliation was significantly related to change in overt ASB across adolescence and early adulthood $(\beta=1.39, z=2.21, p<0.05)$. However, among youth with the high-activity MAOA genotype, deviant peer affiliation was an even stronger predictor of overt ASB $(\beta=2.75, z=9.4, p<0.0001)$. Finally, because deviant peer affiliation may be influenced by MAOA, it was important to test their association. MAOA was unrelated to deviant peer affiliation $(r=0.05, p=0.23)$, thereby reducing concerns about gene-environment correlation (rGE) as an alternative explanation and strengthening the possibility of a potential gene $\times$ environment interaction $(\mathrm{G} \times \mathrm{E})$ for overt ASB.

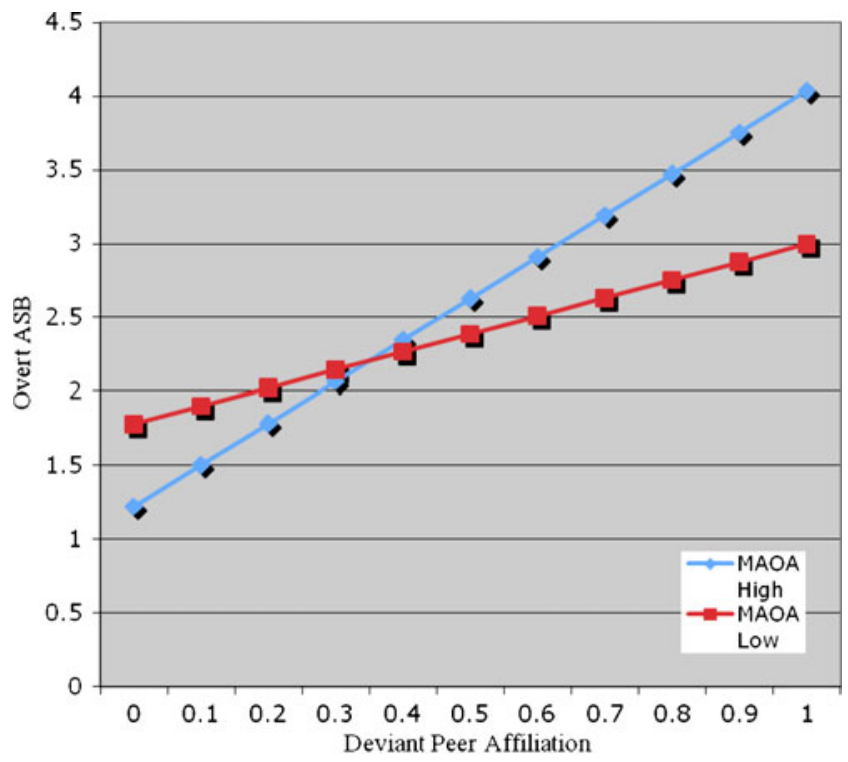

Fig. 1 Overt ASB: MAOA $\times$ deviant peer affiliation interaction 
Covert ASB: MAOA Genotype, Deviant Peer Affiliation, and Their Interaction $(\mathrm{G} \times \mathrm{E})$

Based on 5 covert ASB items, identical models to those tested for overt ASB above were separately tested for covert ASB (see Table 3, Model 1 for model summary consisting of covariates and main effects only). In the fully saturated model, consisting of age, wave, deviant peer affiliation and MAOA genotype main effects, and their interaction (Table 3, Model 2), deviant peer affiliation was significantly related to covert ASB $(\beta=$ 1.88, $z=3.99, p<0.0001)$ where individuals with more extensive deviant peer affiliation reported higher levels of their own covert ASB. However, MAOA was not significantly associated with covert ASB over time $(\beta=-0.11, z=-0.44, p=$ $0.66)$ and the interaction between deviant peer affiliation and MAOA genotype was also nominal $(\beta=0.51, z=0.92, p=$ 0.36) (see Fig. 2).

\section{Discussion}

To address the significant gap in knowledge about potential genetic and environmental influences on ASB, particularly with respect to patterns of ASB over time, data were used from a large sample of Caucasian adolescent boys followed prospectively into early adulthood. This study is the first to incorporate genotype as a moderator of deviant peer affiliation and ASB. The association of the 30-base pair variable number tandem repeat polymorphism of the monoamine oxidase A (MAOA) gene, deviant peer affiliation, and their interaction with self-reported overt and covert ASB across a 6-year period was examined. Consistent with the initial hypothesis, deviant peer affiliation and the low-activity MAOA genotype each predicted overt ASB where youth with the low-activity genotypes

Table 3 Generalized estimating equation (GEE) predicting covert antisocial behavior (ASB)

\begin{tabular}{lccl}
\hline Variables & $\beta$ & $z$ & $p<$ value \\
\hline Model 1 & & & \\
Age & -0.25 & -5.32 & 0.0001 \\
Wave & 0.004 & 0.13 & 0.89 \\
Deviant peer affiliation (DPA) & 2.21 & 9.51 & 0.0001 \\
MAOA genotype & 0.06 & 0.35 & 0.72 \\
Model 2 & & & \\
Age & -0.26 & -5.34 & 0.0001 \\
Wave & 0.004 & -0.13 & 0.89 \\
Deviant peer affiliation (DPA) & 1.88 & 3.99 & 0.0001 \\
MAOA genotype & -0.11 & -0.44 & 0.66 \\
Deviant peer $\times$ MAOA interaction & 0.51 & 0.92 & 0.36 \\
\hline
\end{tabular}

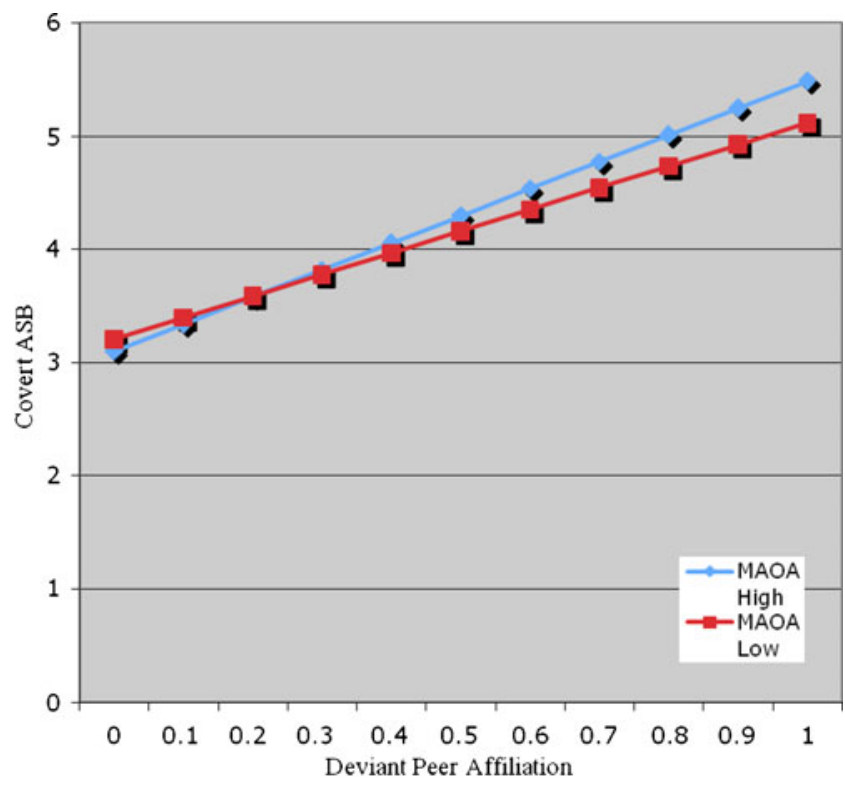

Fig. 2 Covert ASB: MAOA $\times$ deviant peer affiliation interaction

reported significantly more overt ASB than youth with the high-activity genotypes. Deviant peer affiliation also positively predicted levels of overt ASB across development, independent of MAOA, age, and wave. There was evidence suggestive of gene-environment interaction $(\mathrm{G} \times \mathrm{E})$, but its direction was inconsistent with our hypothesis. Deviant peer affiliation positively predicted overt ASB more robustly among youth with the high-activity MAOA genotypes than youth with the low-activity genotypes. Finally, deviant peer affiliation positively predicted covert ASB, but no significant association was observed for MAOA and its interaction with deviant peer affiliation.

Consistent with reports in the literature, individuals in this sample with the low-activity MAOA genotypes reported significantly higher levels of overt ASB across 6 years than youth with high-activity MAOA genotypes. These same lowactivity MAOA genotypes have been implicated in conduct disorder (CD) and criminal behavior (Prom-Wormley et al. 2009; Sjoberg et al. 2007) and substance use disorder (Vanyukov et al. 2007), although null findings have also been reported (Huizinga et al. 2006; Widom and Brzustowick 2006). Sample differences (e.g., treatment seeking vs. population-based), phenotypic definitions (e.g., DSM-IVbased vs. broader measures of ASB), and contrasting approaches to defining the transcriptional efficiency of MAOA polymorphisms (Deckert et al. 1999; Kim-Cohen et al. 2006; Sabol et al. 1998) complicate efforts to directly compare results across studies. Nevertheless, studies of MAOA and endophenotypes related to ASB have illuminated its effects on pathophysiology. Males with the low-activity MAOA genotype had reduced left middle frontal gyrus activation whereas individuals with the high-activity MAOA 
genotype demonstrated heightened activity of neural circuits associated with anger control during an emotional reactivity task (Alia-Klein et al. 2008). The low-activity MAOA genotype was also associated with antisocial personality disorder and alterations in event related potential (ERP) recording during a negative emotion-processing task (Williams et al. 2009). Of particular relevance to the study of ASB and related phenotypes is the fact that in vivo studies of MAOA genotype suggest pleiotropic effects, including amygdala hyper-reactivity to emotional cues and differential activation of caudal regions of the anterior cingulate, which mediate cognitive and inhibitory control (Meyer-Lindenberg et al. 2006). Given the centrality of negative affect and cognitive dimensions of ASB (Lahey 2009; Martel et al. 2007), MAOA remains a candidate gene worthy of further dissection.

The present study extended the search for moderators of deviant peer affiliation and ASB to a functional MAOA variant in an adolescent sample followed into adulthood. Although there is evidence that the high-activity MAOA genotypes are associated with more ASB (Beitchman et al. 2004; van der Vegt et al. 2009), the finding that deviant peer affiliation predicted overt ASB in the high-activity more robustly than in the low-activity genotype contradicted previous studies, although they were based on maltreatment (Caspi et al. 2002; Edwards et al. 2010; Kim-Cohen et al. 2006). Wakschlag et al. (2010) reported sex-specific $G \times E$ results where the low-activity MAOA genotypes interacted with prenatal nicotine exposure in boys, but it was the highactivity MAOA genotypes for girls. Several factors may explain the patterns observed thus far for $G \times E$ studies involving MAOA and ASB. First, research on the development of ASB has prioritized divergent pathways to and from ASB. Loeber (1988) proposed distinct classes of ASB, consisting of exclusive substance use, non-aggressive, and pernicious aggressive/versatile pathways. Defined as equifinality, or multiple pathways eventuating in the same outcome (i.e., ASB) (Cicchetti and Rogosch 1996), these groups were empirically separable, but each was associated with substance use problems (Loeber 1988). Thus, the combination of deviant peer affiliation and high-activity MAOA genotype for overt ASB may represent an empirically distinct group of ASB relative to the 'main effect' of the low-activity MAOA genotype when deviant peer affiliation was controlled. Second, theorists have proposed that the nature of deviant peer influences is not identical across development and ASB subtypes. Lahey et al. (1999) and Moffitt (1993) contend that deviant peer affiliation may indicate latent genetic risk in some youth (i.e., life-coursepersistent; LCP), but play a more direct role for others (i.e., adolescence-limited individuals; AL). Because LCP ASB is more heritable than AL ASB (Moffitt 2003), the stronger effect of deviant peer affiliation in the high-activity group is plausible because deviant peer affiliation may simply be less consequential in the low-activity group. Similarly, the relative contribution and precise role of deviant peer affiliation and ASB in the high-activity genotypes may be environmentally mediated through processes such as peer reinforcement. In the low-activity MAOA genotype, deviant peer affiliation may be secondary to the development of overt ASB because it disrupts neural structures and circuitry, which potentiate violence and related endophenotypes (i.e., negative emotionality) (Buckholtz and Meyer-Lindenberg 2008). Third, given that this sample consisted of adolescents followed into adulthood, the interactive effects between deviant peer affiliation and MAOA must be interpreted within the context of developmental influences. Not only are some forms of adolescent ASB normative (Moffitt 2003), dynamic changes in the organization and connectivity of neural systems related to ASB (e.g., learning, reward sensitivity) (Galvan 2010) must be reconciled in future developmentally-sensitive models of $\mathrm{G} \times \mathrm{E}$. In fact, previous studies of $\mathrm{G} \times \mathrm{E}$ for MAOA and ASB featured different developmental periods and timelines for exposure to environmental risk. Whereas Caspi et al. (2002) assessed maltreatment when participants were between 3 and 11 years-old, physical discipline was assessed prior to age 6 by Edwards et al. (2010), and deviant peer affiliation was assessed at ages $12-20$ in this study.

Interactive models between MAO and environmental risk have emerged, most notably for early maltreatment (Weder et al. 2009; Kim-Cohen et al. 2006), but other forms of adversity are being measured. Wakschlag et al. (2010) found that prenatal nicotine exposure and the low-activity MAOA genotypes predicted ASB in boys, but exposure and the high-activity group predicted ASB in girls. Belsky et al. (2009) re-conceptualized the nature of geneenvironment interplay for disease by emphasizing that susceptibility genes concurrently function as plasticity genes (i.e., contribute to positive outcome) in enriching environments. Unlike diathesis-stress, the plasticity model expects that the low-activity MAOA genotype will predict higher ASB with environmental risk, but lower ASB with environmental enrichment. In addition, deviant peer affiliation may not be purely environmentally mediated, particularly for groups of youth and at particular stages in development, the patterns reported herein are potentially consistent with this formulation. At low levels of deviant peer affiliation, low-activity MAOA genotypes predicted more overt ASB. However, the same low-activity MAOA genotypes were associated with significantly lower levels of overt ASB in the presence of high deviant peer affiliation. Although this theory requires further empirical scrutiny, it challenges assumptions about genetic susceptibility, environmental adversity/nurturance, and the development of psychopathology. 
There are several important implications of this study. Future studies of genetic and environmental influences on psychopathology must transcend the identification of risk factors and risk indicators to identify risk processes. Rutter (2006) argued that genetic influences can be environmentally mediated and environmental effects can be genetically mediated. Thus, designations of genetic and environmental risk do not necessarily convey the actual mechanisms underlying their association with outcome. Identifying these underlying processes should be prioritized in future genetically informative studies. For example, in this study, deviant peer affiliation for the group of children with the high-risk MAOA genotype may simply be a risk indicator rather than a potential causal risk factor for youth without the low-risk genotype. Next, developmental influences are frequently ignored in genetic studies that rely on cross-sectional designs and highly dispersed age ranges. But, prospective longitudinal designs not only improve statistical power, they may also change estimates on the prevalence of psychopathology relative to retrospective designs (Moffitt et al. 2010). These designs also permit informative tests of genotype $\times$ time interactions to ascertain differences in genetic influence over time. This is particularly relevant given that the heritability of ASB changes from childhood to adolescence (Rhee and Waldman 2002). Finally, future studies of $G \times E$ must consider the possibility that different models of $\mathrm{G} \times \mathrm{E}$ and rGE operate with respect to the etiology of disorder from those models of interplay that may maintain or further amplify initial trajectories of disorder.

The current study features several methodological assets including a relatively large sample and a prospective longitudinal design. However, there are several important limitations. First, ASB was measured and analyzed as count data, thereby preventing inferences about diagnostic criteria (e.g., conduct disorder) and functional impairment. It is unclear whether these patterns of association would be preserved across other phenotypic approaches. Future research must adopt alternative methods to phenotypic classification that are developmentally-informed. For example, Odgers et al. (2008) derived four latent classes from the Dunedin sample using growth mixture models that were the basis for hypotheses about the developmental trajectories of offending in men and women. Coupled with prospective longitudinal designs, latent class methods (e.g., latent transition analysis) may discern groups of offenders and potentially identify predictors of continuity. Second, this sample consisted of a wide age range of participants (1220 years). Consequently, deviant peer affiliation cannot be uniformly interpreted across this period. For example, for young adult participants, having peers who smoke and drink is likely to be quite normative relative to adolescents. In fact, when the deviant peer affiliation construct featured in this study excluded the items that assessed peers who smoke and drank (based on the fact that 18-20 year-olds can legally smoke and drink), the interaction with MAOA was no longer significant (results available from author upon request). Thus, peer deviance must be defined in accordance with developmental theories and expectations and perhaps also with socio-cultural standards. Third, Add Health presently consists only of single polymorphisms from five candidate genes. Thus, linkage disequilibrium patterns could not be discerned and genomic control could not be used to address population stratification with the entire sample (i.e., including all racial-ethnic groups) (Weder et al. 2009). Fourth, genome-wide approaches are the emerging standard in genetics, allowing for anonymous interrogation of variation across the entire genome. Because complex phenotypes are explained by numerous genes, each of modest effect size (Risch and Merikangas 1996), traditional candidate gene approaches may progress the field more slowly than genome-wide methods and related innovations (e.g., copy number variation) (Cook and Scherer 2008). Finally, the study does not provide methods to identify potential mediating endophenotypes, particularly those derived from in-vivo (e.g., neuroimaging) assays or psychometrically-sound clinical assessment (e.g., neuropsychological functioning). To advance the field, studies that prioritize repeated measures, diverse genetic and biological assays, and careful phenotypic characterization will improve traction on gene-environment interplaying underlying the development and persistence of ASB.

Acknowledgments This research uses data from the National Longitudinal Study of Adolescent Health (Add Health), a program project designed by J. Richard Udry, Peter S. Bearman, and Kathleen Mullan Harris and funded by the National Institute of Child Health and Human Development Grant PO1-HD31921 with cooperative funding from 17 other agencies. Work on this project was also supported by NIH grant 1R03AA0208186-01 to Steve S. Lee.

Open Access This article is distributed under the terms of the Creative Commons Attribution Noncommercial License which permits any noncommercial use, distribution, and reproduction in any medium, provided the original author(s) and source are credited.

\section{References}

Achenbach, T. M., \& Howell, C. T. (1993). Are American children's problems getting worse? A 13-year comparison. Journal of the American Academy of Child and Adolescent Psychiatry, 32, $1145-1154$.

Alia-Klein, N., Goldstein, R. Z., Kriplani, A., Logan, J., Tomasi, D., Williams, B., et al. (2008). Brain monoamine oxidase A predicts trait aggression. Journal of Neuroscience, 28, 5099-5104.

Beitchman, J. H., Mik, H. M., Ehtesham, S., Douglas, L., \& Kennedy, J. L. (2004). MAOA and persistent, pervasive childhood aggression. Molecular Psychiatry, 9, 546-547. 
Belsky, J., Jonassaint, C., Pleuss, M., Stanton, M., Brummet, B., \& Williams, R. (2009). Vulnerability genes or plasticity genes? Molecular Psychiatry, 14, 746-754.

Brendgen, M., Boivin, M., Vitaro, F., Bukowski, W. M., Dionne, G., Tremblay, R. E., et al. (2008). Linkages between children's and their friends' social and physical aggression: evidence for a geneenvironment interaction? Child Development, 79, 13-29.

Brunner, H., Nelen, M., Breakefield, X., Ropers, H., \& van Oost, B. (1993). Abnormal behavior associated with a point mutation in the structural gene for monoamine oxidase A. Science, 262, 578580 .

Buckholtz, J. W., \& Meyer-Lindenberg, A. (2008). MAOA and the neurogenetic architecture of human aggression. Trends in Neuroscience, 30, 120-129.

Buckholtz, J. W., Callicott, J. H., Kolachana, B., Hariri, A. R., Goldberg, T. E., Genderson, M. et al. (2008). Genetic variation in MAOA modulates ventromedial prefrontal circuitry mediating individual differences in human personality. Molecular Psychiatry, 13,313-324.

Burt, A. (2009). Are there meaningful etiological differences within antisocial behavior? Results of a meta-analysis. Clinical Psychology Review, 29, 163-178.

Carrel, L., \& Willard, H. (2005). X-inactivation profile reveals extensive variability in $\mathrm{X}$-linked gene expression in females. Nature, 434, 400-404.

Cases, O., Seif, I., Grimsby, J., Gaspar, P., Chen, K., Pournin, S., et al. (1995). Aggressive behavior and altered amounts of brain serotonin and norepinephrine in mice lacking MAOA. Science, $268,1763-1766$

Caspi, A., McClay, J., Moffitt, T. E., Mill, J., Martin, J., Craig, I. W., et al. (2002). Role of genotype in the cycle of violence in maltreated children. Science, 297, 851-854.

Cicchetti, D., \& Rogosch, F. A. (1996). Equifinality and multifinality in developmental psychopathology. Development and Psychopathology, 8, 597-600.

Cleveland, H. H., Wiebe, R. P., Rowe, D. C. (2005). Sources of exposure to smoking and drinking friends among adolescents: a behavioral genetic evaluation. Journal of Genetic Psychology, 166, 153-169.

Cook, E. H., \& Scherer, S. W. (2008). Copy-number variations associated with neuropsychiatric conditions. Nature, 455, 919-923.

Deckert, J., Catalano, M., Syagailo, Y., Okladnova, O., DiBella, D., Nothen, M., et al. (1999). Excess of high-activity of monoamine oxidase A gene promoter alleles in female patients with panic disorder. Human Molecular Genetics, 8, 621-624.

Dishion, T. J., \& Patterson, G. R. (2006). The development and ecology of antisocial behavior in children and adolescents. In D. Cicchetti \& D. J Cohen (Eds.), Developmental psychopathology (2nd ed., pp. 503-541). New York: Wiley.

Dodge, K. A., Lansford, J. E., \& Dishion, T. J. (2006). The problem of deviant peer influences in intervention programs. In $\mathrm{K}$. A. Dodge, T. J. Dishion, \& J. E. Lansford (Eds.), Deviant peer influences in programs for youth (pp. 3-13). New York: Guilford.

Edelbrock, C., Rende, R., Plomin, R., \& Thompson, L. A. (1995). A twin study of competence and problem behavior in childhood and early adolescence. Journal of Child Psychology and Psychiatry, 36, 775-785.

Edwards, A. C., Dodge, K. A., Latendresse, S. J., Lansford, J. E., Bates, J. E., Pettit, G. E., et al. (2010). MAOA-uVNTR and early physical discipline interact to influence delinquent behavior. Journal of Child Psychology and Psychiatry, 51, 679-687.

Fergusson, D. M., Swain-Campbell, N. R., \& Horwood, L. J. (2002). Deviant peer affiliations, crime, and substance use: a fixed regression analysis. Journal of Abnormal Child Psychology, 30, 419-430.
Foley, D. L., Eaves, L. J., Wormley, B., Silberg, J. L., Maes, H. H., Kuhn, J., et al. (2004). Childhood adversity, monoamine oxidase a genotype, and risk for conduct disorder. Archives of General Psychiatry, 61, 738-744.

Frick, P. J., Lahey, B. B., Loeber, R., Tannenbaum, L., Van Horn, Y., Christ, M. A. G., et al. (1993). Oppositional defiant disorder and conduct disorder: a meta-analytic review of factor analyses and cross-validation in a clinic sample. Clinical Psychology Review, 13(4), 319-340.

Galvan, A. (2010). Adolescent development of the reward system. Frontiers in Human Neuroscience, 4, 1-9.

Gardner, T. W., Dishion, T. J., \& Connell, A. M. (2004). Adolescent self-regulation as resilience: resistance to antisocial behavior within the deviant peer context. Journal of Abnormal Child Psychology, 36, 273-284.

Goodman, S. H., Lahey, B. B., Fielding, B., Dulcan, M., Narrow, W., \& Regier, D. (1997). Representativeness of clinical samples of youths with mental disorders: a preliminary population-based study. Journal of Abnormal Psychology, 106, 3-14.

Goodnight, J. A., Bates, J. E., Newman, J. P., Dodge, K. A., \& Pettit, G. S. (2006). The interactive influences of friend deviant and reward dominance on the development of externalizing behavior during middle adolescence. Journal of Abnormal Child Psychology, 34, 573-583.

Gordon, R. A., Lahey, B. B., Kawai, E., Loeber, R., Stouthamer-Loeber, M., \& Farrington, D. P. (2004). Antisocial behavior and gang membership: selection and socialization. Criminology, 42, 55-87.

Gottesman, I. I., \& Gould, T. D. (2003). The endophenotype concept in psychiatry: etymology and strategic intentions. American Journal of Psychiatry, 160, 636-645.

Haberstick, B. C., Lessem, J. M., Hopfer, C. J., Smolen, A., Timberlake, D., \& Hewitt, J. K. (2005). Monoamine oxidase A (MAOA) genotype and antisocial behaviors in childhood and adolescent maltreatment. American Journal of Medical Genetics Part B: Neuropsychiatric Genetics, 135, 59-64.

Hanley, J. A., Negassa, A., Edwardes, M. D., \& Forrester, J. E. (2003). Statistical analysis of correlated data using generalized estimating equations: an orientation. American Journal of Epidemiology, 157, 364-375.

Hinshaw, S. P., \& Lee, S. S. (2003). Conduct and oppositional defiant disorders. In E. J. Mash \& R. A. Barkley (Eds.), Child psychopathology (2nd ed., pp. 144-198). New York: Guilford.

Hinshaw, S. P., Simmel, C., \& Heller, T. (1995). Multimethod assessment of covert antisocial behavior in children: laboratory observations, adult ratings, and child self-report. Psychological Assessment, 7, 207-219.

Huizinga, D., Haberstick, B. C., Smolen, A., Menard, S., Young, S. E., Corley, R. P., et al. (2006). Childhood maltreatment, subsequent antisocial behavior, and the role of monoamine oxidase A genotype. Biological Psychiatry, 60, 677-683.

Hutchison, K. E., Stallings, M., McGeary, J. M., \& Bryan, A. (2004). Population stratification in the case-control design: fatal threat or red herring? Psychological Bulletin, 130(1), 66-79.

Jaffee, S. R., \& Price, T. S. (2007). Gene-environment correlations: a review of the evidence and implications for prevention of mental illness. Molecular Psychiatry, 12, 432-442.

Karere, G. M., Kinnally, E. L., Sanchez, J. N., Famula, T. R., Lyons, L. A., \& Capitanio, J. P. (2009). What is an "adverse" environment? Interactions of rearing experiences and MAOA genotype in rhesus monkeys. Biological Psychiatry, 65, 770-777.

Kazdin, A. E. (1995). Conduct disorder in childhood and adolescence (2nd ed.). Thousand Oaks: Sage.

Keenan, K., Wroblewski, K., Hipwell, A., Loeber, R., \& Stouthamer-Loeber, M. (2010). Age of onset, symptom threshold, and expansion of the nosology of conduct disorder for girls. Journal of Abnormal Psychology, 119 689-698. 
Kendler, K. S., Jacobson, K. C., Gardner, C. O., Gillespie, N., Aggen, S. A., \& Prescott, C. A. (2007). Creating a social world: a developmental twin study of peer-group deviance. Archives of General Psychiatry, 64, 958-965.

Kendler, K. S., Jacobson, K. C., Myers, J. M., \& Eaves, L. J. (2008). A genetically informative study of the relationship between conduct disorder and peer deviance in males. Psychological Medicine, 38, 1001-1011.

Kim-Cohen, J., Caspi, A., Taylor, A., Williams, B., Newcombe, R., Craig, I. W., et al. (2006). MAOA, maltreatment, and geneenvironment interaction predicting children's mental health: new evidence and a meta-analysis. Molecular Psychiatry, 11, 903-913.

Lacourse, E., Nagin, D. S., Vitaro, F., Cote, S., Arsenault, L., \& Tremblay, R. E. (2006). Prediction of early-onset deviant peer group affiliation: a 12-year longitudinal study. Archives of General Psychiatry, 63, 562-568.

Lahey, B. B. (2009). Public health significance of neuroticism. American Psychologist, 64, 241-256.

Lahey, B. B., Waldman, I. D., \& McBurnett, K. (1999). Annotation: the development of antisocial behavior: an integrative causal model. Journal of Child Psychology and Psychiatry, 40, 669682.

Lahey, B. B., Loeber, R., Burke, J. D., Rathouz, P., \& McBurnett, K. (2002). Waxing and waning in concert: dynamic comorbidity of conduct disorder with other disruptive and emotional problems over seven years among clinic-referred boys. Journal of Abnormal Psychology, 111, 556-567.

Lee, S. S., \& Hinshaw, S. P. (2004). Severity of adolescent delinquency among boys with and without attention-deficit hyperactivity disorder (ADHD): predictions from early antisocial behavior and peer status. Journal of Clinical Child and Adolescent Psychology, 33, 705-716.

Lee, S. S., \& Hinshaw, S. P. (2006). Predictors of adolescent functioning in girls with attention-deficit/hyperactivity disorder (ADHD): the role of childhood ADHD, conduct problems, and peer status. Journal of Clinical Child and Adolescent Psychology, 35, 356-368.

Li, J. J., \& Lee, S. S. (2010). Latent class analysis of antisocial behavior: interaction of serotonin transporter genotype and maltreatment. Journal of Abnormal Child Psychology, 38, 789-801.

Loeber, R. (1988). Natural histories of conduct problems, delinquency, and associated substance use: Evidence for developmental progressions. In B. B. Lahey \& A. E. Kazdin (Eds.), Advances in clinical child psychology (pp. 73-124). New York: Plenum.

Loeber, R., \& Stouthamer-Loeber, M. (1998). The development of juvenile aggression and violence: Some common misconceptions and controversies. American Psychologist, 53, 242-259.

Loeber, R., Farrington, D. P., Stouthamer-Loeber, M., \& Van Kammen, W. B. (1998). Antisocial behavior and mental health problems: Explanatory factors in childhood and adolescence. Mahwah: Erlbaum.

Martel, M. M., Nigg, J. T., Wong, M. M., Fitzgerald, H. E., Jester, J. M., Puttler, L. I., et al. (2007). Childhood and adolescent resiliency, regulation, and executive functioning in relation to adolescent problems and competence in a high-risk sample. Development and Psychopathology, 19, 541-563.

Meyer-Lindenberg, A., Buckholtz, J. W., Kolachana, B., Harriri, A. R., Pezawas, L., Blasi, G., et al. (2006). Neural mechanisms of genetic risk for impulsivity and violence in humans. Proceedings of the National Academy of Sciences, 103, 6269-6274.

Moffitt, T. E. (1993). "Life-course persistent" and "adolescencelimited" antisocial behavior: a developmental taxonomy. Psychological Review, 100, 674-701.

Moffitt, T. E. (2003). Life-course-persistent and adolescence-limited antisocial behavior: A 10-year research review and a research agenda. In B. B. Lahey, T. E. Moffitt, \& A. Caspi (Eds.), Causes of conduct disorder and juvenile delinquency (pp. 49-75). New York: Guilford.

Moffitt, T. E., Caspi, A., Rutter, M., \& Silva, P. (2001). Sex differences in antisocial behavior: Conduct disorder, delinquency, and violence in the Dunedin longitudinal study. Cambridge: Cambridge University Press.

Moffitt, T. E., Caspi, A., Taylor, A., Kokaua, J., Milne, B. J., Polanczyk, G., et al. (2010). How common are mental disorders? Evidence that lifetime prevalence rates are doubled by prospective versus retrospective ascertainment. Psychological Medicine, 40, 899-909.

Monahan, K. C., Steinberg, L., \& Cauffman, E. (2009). Affiliation with antisocial peers, susceptibility to peer influence, and antisocial behavior during the transition to adulthood. Developmental Psychology, 45, 1520-1530.

Monuteaux, M. C., Biederman, J., Doyle, A. E., Mick, E., \& Faraone, S. V. (2009). Genetic risk for conduct disorder symptom subtypes in an ADHD sample: specificity to aggressive symptoms. Journal of the American Academy of Child and Adolescent Psychiatry, 48, 757-764.

Odgers, C. L., Moffitt, T. E., Broadbent, J. M., Dickson, N., Hancox, R. J., Harrington, H., et al. (2008). Female and male antisocial trajectories: from childhood origins to adult outcomes. Development and Psychopathology, 20, 673-716.

Ozelius, L., Hsu, Y. P., Bruns, G., Powell, J. F., Chen, S., Weyler, W., et al. (1988). Human monoamine oxidase gene (MAOA): chromosome position (Xp21-p11) and DNA polymorphism. Genomics, 3, 53-38.

Prom-Wormley, E. C., Eaves, L. J., Foley, D. L., Gardner, C. O., Archer, K. J., Wormley, B. K., et al. (2009). Monoamine oxidase $\mathrm{A}$ and childhood adversity as risk factors for conduct disorder in females. Psychological Medicine, 39, 579-590.

Rhee, S. H., \& Waldman, I. D. (2002). Genetic and environmental influences on antisocial behavior: a meta-analysis of twin and adoption studies. Psychological Bulletin, 128, 490-529.

Risch, N., \& Merikangas, K. (1996). The future of genetic studies of complex human diseases. Science, 273, 1516-1517.

Rutter, M. (2006). Genes and behavior: Nature-nurture interplay explained. Malden: Blackwell.

Sabol, S. Z., Hu, S., \& Hamer, D. (1998). A functional polymorphism in the monoamine oxidase A gene promoter. Human Genetics, 103, 273-279.

Schmitz, S., Cherny, S. S., \& Fulker, D. W. (1998). Increase in power through multivariate analyses. Behavior Genetics, 28, 357-363.

Sjoberg, R. L., Nilsson, K. W., Wargelius, H., Leppert, J., Lindstrom, L., \& Oreland, L. (2007). Adolescent girls and criminal activity: role of MAOA-LPR genotype and psychosocial factors. American Journal of Medical Genetics Part B: Neuropsychiatric Genetics, 144B, 159-164.

Snyder, J., Schrepferman, L., Oeser, J., Patterson, G., Stoolmiller, M., Johnson, K., et al. (2005). Deviancy training and association with deviant peers in young children: occurrence and contribution to early-onset conduct problems. Development and Psychopathology, 17, 397-413.

van der Vegt, E. J., Oostra, B. A., Arias-Vasquez, A., van der Ende, J., Verhulst, F. C., \& Tiemeier, H. (2009). High-activity of Monoamine oxidase $\mathrm{A}$ is associated with externalizing behavior in maltreated and nonmaltreated adoptees. Psychiatric Genetics, 19, 209-211.

van Lier, P., Boivin, M., Dionne, G., Vitaro, F., Brendgen, M., Koot, H., et al. (2007). Kindergarten children's genetic vulnerabilities interact with friends' aggression to promote children's own aggression. Journal of the American Academy of Child and Adolescent Psychiatry, 46, 1080-1087.

Vanyukov, M. M., Maher, B. S., Devlin, B., Kirillova, G. P., Kirisci, L., Yu, L. M., et al. (2007). The MAOA promoter polymorphism, disruptive behavior disorders, and early onset substance use 
disorder: gene-environment interaction. Psychiatric Genetics, 17, 323-332.

Vitaro, F., Brendgen, M., \& Tremblay, R. E. (2000). Influence of deviant friends on delinquency: search for moderator variables. Journal of Abnormal Child Psychology, 28, 313-325.

Wakschlag, L. S., Kistner, E. O., Pine, D. S., Biesecker, G., Pickett, K. E., Skol, A. D., et al. (2010). Interaction of prenatal exposure to cigarettes and MAOA genotype in pathways to youth antisocial behavior. Molecular Psychiatry, 15, 928-937.

Weder, N., Yang, B. Z., Douglas-Palumberi, H., Massey, J., Krystal, J. H., Gelernter, J., et al. (2009). MAOA genotype, maltreatment, and aggressive behavior: the changing impact of genotype at varying levels of trauma. Biological Psychiatry, 65, 417-424.

Widom, C. S., \& Brzustowick, L. M. (2006). MAOA and the "cycle of violence:" childhood abuse and neglect, MAOA genotype, and risk for violent and antisocial behavior. Biological Psychiatry, 60, 684-689.

Williams, L. M., Gatt, J. M., Kuan, S. A., Dobston-Stone, C., Palmer, D. M., Paul, R. H., et al. (2009). A polymorphism of the MAOA gene is associated with emotional brain markers and personality traits on an antisocial index. Neuropsychopharmacology, 34, 1797-1809.

Willoughby, M., Kupersmidt, J., \& Bryant, D. (2001). Overt and covert dimensions of antisocial behavior in early childhood. Journal of Abnormal Child Psychology, 29(3), 177-187.

Young, S. E., Smolen, A., Hewitt, J. K., Haberstick, B. C., Stallings, M. C., Corley, R. P., et al. (2006). Interaction between MAO-A genotype and maltreatment in the risk for conduct disorder: failure to confirm in adolescent patients. American Journal of Psychiatry, 163, 1019-1025. 